\title{
The isoperimetric spectrum of finitely presented groups
}

\author{
M.V. Sapir ${ }^{*}$
}

\begin{abstract}
The isoperimeric spectrum consists of all real positive numbers $\alpha$ such that $O\left(n^{\alpha}\right)$ is the Dehn function of a finitely presented group. In this note we show how a recent result of Olshanskii completes the description of the isoperimetric spectrum modulo the celebrated Computer Science conjecture (and one of the seven Millennium Problems) $\mathbf{P}=\mathbf{N P}$ and even a formally weaker conjecture.
\end{abstract}

The goal of this note is to show that the recent paper by Olshanskii [7] completes a description of the isoperimetric spectrum of finitely presented groups modulo the $\mathbf{P}=\mathbf{N P}$ conjecture.

Since in this note we consider only polynomially bounded functions $\mathbb{N} \rightarrow \mathbb{R}$, we call two functions $f, g$ equivalent if $a f(n) \leq g(n) \leq b f(b)$ for some positive constants $a, b$.

Recall that Brady and Bridson [5] called the set of all real numbers $\alpha \geq 1$ such that $n^{\alpha}$ is equivalent to the Dehn function of a finitely presented group the isoperimetric spectrum. When it was introduced, it was known only that all natural numbers belong to the isoperimetric spectrum (the free nilpotent group of class $c$ with at least 2 generators has Dehn function $n^{c+1}[1]$ ), and that by Gromov's theorem the intersection of the isoperimetric spectrum with the open interval $(1,2)$ is empty. It is obvious also that the isoperimetric spectrum is a countable set since the set of all finite group presentation is countable. Bridson [5] found the first examples of non-integral numbers in the spectrum.

Soon after, we proved in [10] that for $\alpha \geq 4$ to be in the isoperimetric spectrum, it is enough that $\alpha$ is computed in time $\leq 2^{2^{m}}$. Recall [10] that a real number $\alpha$ is called computable in time $T(m)$ where $T(m)$ is a function $\mathbb{N} \rightarrow \mathbb{N}$, if there exists a deterministic Turing machine which, given a natural number $n$, computes a binary rational approximation of $\alpha$ with an error at most $1 / 2^{n+1}$ in at most $T(n)$ steps. Thus all algebraic numbers $\geq 4$ and many transcendental numbers such as $\pi+1$ are in the isoperimetric spectrum. On the other hand, we proved in [10] that every number in the isoperimetric spectrum can be computed in time $\leq 2^{2^{2^{c m}}}$ for some constant $c$. It can be seen from the proof of this result that the number of 2's in this estimate can be reduced to two as in the lower bound if we had $\mathbf{P}=\mathbf{N P}$. I mentioned it (without a proof) in my ICM talk [8] and my Bulletin of Mathematical Sciences survey [9].

For $\alpha \leq 4$, the situation was more complicated. On the one hand the tools used in [10] were too weak to handle $\alpha \leq 4$. On the other hand, Brady, Bridson, Forester and Shankar found more numbers from the interval $(2,4)$ in the isoperimetric spectrum, showing that the set of these numbers is dense in the interval $(2,4)[2$ and even contains all rational numbers [3]. Their numbers from the isioperimetric spectrum were constructed using

\footnotetext{
${ }^{*}$ Supported in part by the NSF grant DMS-1500180.

${ }^{\dagger}$ Key words: Dehn function, finitely presented group, isoperimetric spectrum, $\mathrm{P}=\mathrm{NP}$

${ }^{\ddagger}$ AMS Mathematical Subject Classification: 20F05, 20F06, 20F65, 20F69, 03D10.
} 
algebraic rather than computational properties. (Note also that the groups constructed in [5, 2, 4, 3], are given by very small presentations comparing to the groups in [10] and are subgroups of $\mathrm{CAT}(0)$ groups which is quite remarkable.) But the paper by Olshanskii [7] showed that the intersection of the isoperimetric spectrum with $(2,4)$ can be described in the same terms as in [10].

Combining results of [10] and [7] we get:

Theorem 1 (The first part of Corollary 1.4 from [10], and Corollary 1.4 from [7]). If a number $\alpha \geq 2$ can be computed in time $\leq 2^{c 2^{m}}$ for some $c$, then $\alpha$ belongs to the isoperimetric spectrum.

Notice that one needs to modify a little the proof of the first part of Corollary 1.4 from [10] to obtain the estimate $\leq 2^{c 2^{m}}$ instead of $\leq 2^{2^{m}}$. For this, one should take natural number $d>\log _{2} c$ which is a power $2^{k}$ for some $k$ and consider $d$-ary representations of numbers instead of binary representations as in [10]. Each $d$-ary digit of $\alpha$ is $k$ binary digits. So if the first $m$ binary digits of $\alpha$ are computed in time $\leq 2^{c 2^{m}}$, then the first $m d$-ary digits of $\alpha$ are computed in time at most $d^{d^{m}}$ and the rest of the proof of 10 , Corollary 1.4] carries by replacing 2 by $d$ everywhere.

Now we will prove the main result of the note.

Theorem 2. Provided $\mathbf{P}=\mathbf{N P}$, a number $\alpha$ is in the isoperimetric spectrum if and only if it can be computed in time $\leq 2^{c 2^{m}}$ for some $c \geq 1$.

Proof. Theorem 1 gives one part of Theorem 2.

To prove the other part, suppose that $n^{\alpha}$ is the Dehn function of a finitely presented group. Then by [10, Theorem 1.1], $n^{\alpha}$ is equivalent to the time function $T(n)$ of some (non-deterministic) Turing machine $M$ which recognizes the word problem in a finitely presented group.

As explained by Emil Jeřábek [6], the following property follows from $\mathbf{P}=\mathbf{N P}$ :

$\left.{ }^{*}\right)$ There is a deterministic Turing machine $M^{\prime}$ computing a function $T^{\prime}(n)$ which is equivalent to $T(n)$ and having time function at most $T(n)^{d}$ for some constant $d$.

Since $\alpha \geq 2$, for any $n>0$

$$
\epsilon_{1} n^{\alpha} \leq T^{\prime}(n) \leq \epsilon_{2} n^{\alpha}
$$

for some positive constants $\epsilon_{1} \leq 1$ and $\epsilon_{2} \geq 1$. Let number $n_{0}$ be such that $2^{n}>$ $\log _{2}\left(\epsilon_{2} / \epsilon_{1}\right)$ for every $n \geq n_{0}$. Let $q=[\alpha]+1$.

Given this machine $M^{\prime}$ with, say, $k$ tapes, consider the following Turing machine $M^{\prime \prime}$ which will calculate the first $m$ digits of $\alpha$ (for every $m$ ). This machine has $k+3$ tapes with tape $k+3$ being the input tape. It starts with number $m$ in binary written on tape $k+3$ and all other tapes empty. Then it calculates the number $n=2^{2^{m+n_{0}}}$ and writes it on tape $k+1$ (using tape $k+2$ as an auxiliary tape and cleaninig it after $n$ is computed). Then $M^{\prime \prime}$ turns on the machine $M^{\prime}$ and produces $T^{\prime}(n)$ on tape $k+2$. Then it calculates $p=\left[\left(\log _{2} T^{\prime}(n)-\log _{2} \epsilon_{1}\right) / 2^{n_{0}}\right]$ and writes it on tape $k+2$. Notice that $\alpha \log _{2} n+\log _{2} \epsilon_{1} \leq \log _{2} T^{\prime}(n) \leq \alpha \log _{2} n+\log _{2} \epsilon_{2}$. Therefore

$$
\left[\alpha 2^{m}\right] \leq p \leq \alpha 2^{m}+\log _{2}\left(\epsilon_{2} / \epsilon_{1}\right) / 2^{n_{0}} .
$$


Hence $p=\left[\alpha 2^{m}\right]$, so $p / 2^{m}$ a rational approximation of $\alpha$ which is within $1 / 2^{m}$ from $\alpha$. From the construction of $M^{\prime \prime}$, it is clear that the time complexity of $M^{\prime \prime}$ does not exceed $2^{c 2^{m}}$ for some constant $c$.

As explained by Emil Jeřábek [6], Property (*) follows also from the property $\mathbf{E}=\mathbf{\Sigma}_{\mathbf{2}}^{\mathbf{E}}$, which is not known to imply $\mathbf{P}=\mathbf{N P}$. Here $\mathbf{E}$ denotes the class of languages recognized by deterministic Turing machines in time $2^{O(n)}$ and $\boldsymbol{\Sigma}_{\mathbf{2}}^{\mathbf{E}}$ is the second level of the exponential hierarchy (with linear exponent). Thus in Theorem 2, one can replace $\mathbf{P}=\mathbf{N P}$ by $\mathbf{E}=\boldsymbol{\Sigma}_{\mathbf{2}}^{\mathbf{E}}$.

Acknowledgement. I am grateful to Emil Jeřábek for answering my question [6] and several useful remarks. I am also grateful to Jean-Camille Birget and A. Yu. Olshanskii for their comments.

\section{References}

[1] G. Baumslag, C. F. Miller III, and H. Short, Isoperimetric inequalities and the homology of groups, Invent. Math. 113 (1993), 531-560.

[2] N. Brady and M. Bridson, There is only one gap in the isoperimetric spectrum, Geometric and Functional Analysis, 10 (2000), 1053-1070.

[3] Noel Brady, Martin R. Bridson, Max Forester, Krishnan Shankar, Snowflake groups, Perron-Frobenius eigenvalues and isoperimetric spectra. Geom. Topol. 13 (2009), no. $1,141-187$.

[4] Noel Brady, Max Forester, Snowflake geometry in CAT(0) groups. J. Topol. 10 (2017), no. 4, 883-920.

[5] M. Bridson, Fractional isoperimetric inequalities and subgroup distortion. J. Amer. Math. Soc. 12 (1999), no. 4, 1103-1118.

[6] Mathoverflow, question 307629.

[7] A. Yu. Olshanskii, Polynomially-bounded Dehn functions of groups, arXiv:1710.00550, accepted in Journal of Combinatorial Algebra, 2018.

[8] M. V. Sapir, Algorithmic and asymptotic properties of groups. International Congress of Mathematicians. Vol. II, 223-244, Eur. Math. Soc., Zürich, 2006.

[9] M. V. Sapir, Asymptotic invariants, complexity of groups and related problems. Bull. Math. Sci. 1 (2011), no. 2, 277-364.

[10] M. V. Sapir, J. C. Birget, E. Rips, Isoperimetric and isodiametric functions of groups, Annals of Mathematics, 157, 2(2002), 345-466. 